\title{
Sensor Fusion in Analogue Circuit Fault Diagnosis Using Transferable Belief Model
}

\author{
Youhui Chen, Dong Cheng, Yimin Yang, Zan Xiao \\ College of Electrical and Information Engineering \\ Hunan University \\ Changsha, China \\ shuteoliver@163.com, chengdongchina@163.com, lenck@163.com,xiaozan0606@163.com
}

\begin{abstract}
In fault diagnosis of circuit, fault diagnosis system using multi-sensor data fusion may not give reliable fault diagnosis result in cases when processing on inconsistent data carry inconsistent. In order to deal with such problems, This paper has developed an analog-circuit fault diagnostic system based on transferable belief model (TBM) and K-nearest neighbor rule from TBM. The proposed system has the capability to detect and identify fault components in an analog electronic circuit by analyzing its working temperature and testable voltage. Using $\mathrm{K}$-nearest neighbor(KNN) rule to process the working temperature of component drastically enhances the testable information for fault detect, satisfying the need of fusing data from distinct evidence sources. The experimental results show that this system performs significantly better in fault diagnosis of analog circuits due to the proposed techniques.
\end{abstract}

Keywords-analog circuit; fault diagnosis; transferable belief model; k-nearest neighbor rule from TBM; fault location; data fusion

\section{INTRODUCTION}

Analog fault diagnosis has been an active area of research since the mid-1970s with significant work carried out at the system, board, and chip level[1]-[3], and it remains crucial, particularly for rapidly developed mixedmode(analog and digital) circuits.

While, multi-sensor data fusion system is an important component in many fields dealing with pattern recognition, identification and diagnosis for fault location. It is used with the hope that the aggregation of several sensors from testing different fault information of analog circuits achieves better results. Because of this, many works have already applied information fusion to signal process, engine fault diagnosis [4]-[5], fault diagnosis in circuit. The transferable belief model (TBM) is a model for the quantified representation of epistemic uncertainty and which can be a robot, an intelligent sensor, etc, and provides a highly flexible model to manage the uncertainty encountered in the multi-sensor data fusion problems.

In the TBM framework, two main families of classifiers have also been defined [6]:

- The TBM model-based classifier developed by Smets based on the general Bayesian theorem, the extension of Bayes' theorem in the TBM framework.

- The TBM cased-based classifier developed by Denoeux and Smets, a nonparametric method akin to statistical cased-based classifiers such as the Knearest neighbor classification rule.

The goal of this paper is to present a way to diagnose faulty component in analogues circuits based on transferable belief model. The diagnosing results show that this method can reduce the uncertainty of analog electronic components fault diagnosis, and exactly recognize the fault components.

\section{TRANSFERABLE Belief Model（TBM）}

In this section, we briefly regroup some basic of the belief function theory as explained in the transferable belief model (TBM). More details can be found in [7]-[8].

\section{TBM characteristics}

TBM produces a quantitative degree of belief for each of the hypotheses from a set of all hypotheses $\Omega$ (called the frame of discernment). In TBM, $\Omega$ consists of the three subsets:

- A set of hypotheses known as possible (PH)

- A sent of hypotheses known as impossible (IH)

- A set of unknown hypotheses (UH)

As soon as new evidence becomes available, hypotheses are redistributed among the three sets:

- A hypothesis A is transferred from $\mathrm{PH}$ to $\mathrm{IH}$ if the evidence claims it is impossible;

- A hypothesis A is transferred from $\mathrm{UH}$ to $\mathrm{PH}$ if the evidence suggests that it could be considered possible;

- A hypothesis A is transferred from UH to IH if the evidence suggests that it is impossible.

\section{APPLICATION OF TBM TO FAULT DIAGNOSIS OF ANALOGUE CIRCUITS}

We show here that the fault diagnosis problem of circuits admits a similar solution when uncertainty is quantified by belief function, as in the transferable belief model. When applying the transferable belief model to multi-sensor information fusion, data obtained from sensor is the theory's evidence, and it constitutes the belief function assignment of the object mode needed to be tested, represents a reliable degree of each hypothesis object mode, and each sensor forms an evident group. Multi-sensor information fusion amounts to combining several evident groups to form a new comprehensive evident group. That is to say, we can use the transferable belief model to produce 
comprehensive and precise information for judging object mode.

In this section, we use of TBM from section II and KNN classifier based on TBM framework which mentioned below for fault diagnosis of circuit. Flow chart of proposed fault detection method is shown in Fig.1. Two sensors measure accessible node voltage and temperature of component respectively. The voltage of each accessible node is obtained by using probes, the temperature of each component is measured by using a thermal image instrument.

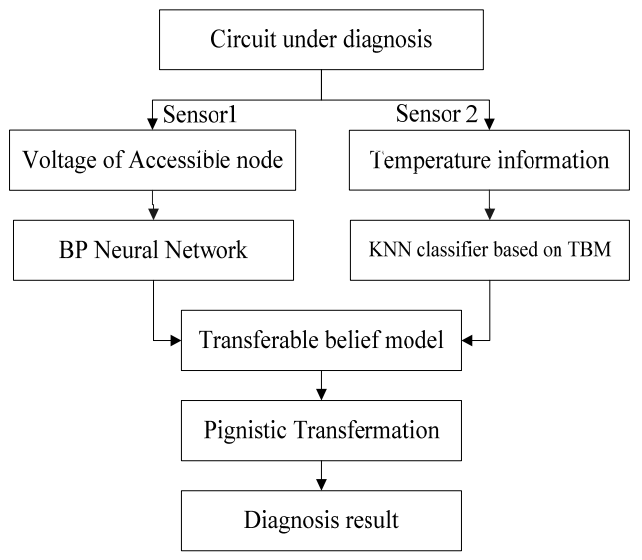

Fig.1 Flow chart of proposed fault detection method

\section{A. K-nearest neighbors classifiers based on TBM framework}

This method was originally introduced in [9]. It consists of considering each example of the training set as an item of evidence rewarding the class membership of a new vector to be classified. The strength of this evidence is assumed to depend on a measure of distance between feature vectors, which is accounted for by discounting each item of evidence with a discount rate defined as a function of distance. A decision is made by assigning a pattern to the class with the maximum credibility by pignistic in TBM.

Let $\Omega=\left\{w_{1}, w_{2}, \ldots, w_{M}\right\}$ denote the $M$ classes. Considering $X$ to be classified and $\psi_{\mathrm{k}}$ is the set of its $\mathrm{K}$ nearest neighbors in a training set $T$. For any $X^{\mathrm{i}} \in \Psi_{\mathrm{k}}$, the knowledge that $\mathrm{X}^{\mathrm{i}}$ belongs to class $\mathrm{w}_{\mathrm{q}}\left(\mathrm{w}_{\mathrm{q}} \in \Omega\right)$ can be considered as a piece of evidence that increases our belief that $\mathrm{X}$ also belongs to $\mathrm{w}_{\mathrm{q}}$. This item of evidence can be represented by a BBA $m_{i}$ assigning a fraction $\partial_{q}^{i}$ of the unit mass to the singleton $\left\{w_{q}\right\}$ and the rest to $\Omega$.

$$
\begin{aligned}
& \mathrm{m}^{\mathrm{i}}\left(\left\{\mathrm{w}_{\mathrm{q}}\right\}\right)=\partial_{\mathrm{q}}^{\mathrm{i}} \\
& \mathrm{m}^{\mathrm{i}}(\Omega)=1-\partial_{\mathrm{q}}^{\mathrm{i}}
\end{aligned}
$$

With $\mathrm{m}^{\mathrm{i}}(\mathrm{A})=0$ for all $\mathrm{A} \subseteq \Omega$ and $\mathrm{A} \notin\left\{\Omega,\left\{\mathrm{w}_{\mathrm{q}}\right\}\right\}$. The mass $\partial_{\mathrm{q}}^{\mathrm{i}}$ is chosen as a decreasing function of Euclidean distance $\mathrm{d}^{\mathrm{i}}$ between $\mathrm{X}$ and $\mathrm{X}^{\mathrm{i}}$.

$$
\partial_{\mathrm{q}}^{\mathrm{i}}=\partial_{0} \varphi_{0}\left(\mathrm{~d}^{\mathrm{i}}\right)
$$

Where the index qindicates that the influence of $\mathrm{d}^{\mathrm{s}, \mathrm{i}}$ may depend on the class of $x^{s}$, the following additional conditions must be imposed on $\partial_{0}$ and $\varphi_{0}$ :

$$
\begin{array}{ll}
\text { st: } & 0<\alpha_{0}<1 \\
& \phi_{\mathrm{q}}(0)=1 \\
\lim \quad{ }_{\mathrm{d} \rightarrow \infty} \phi_{\mathrm{q}}(\mathrm{d})=0
\end{array}
$$

We choose as:

$$
\partial_{q}^{i}=\partial_{0} \exp \left(-\gamma_{q}^{2}\left(d^{2}\right)^{2}\right)
$$

Where $\gamma_{q}^{2}$ a parameter is associated to class $w_{q}$ and $\partial_{0}$ fixed parameter. The BBAs $m^{1}, m^{2}, \ldots, m^{k}$ corresponding to the $\mathrm{K}$-nearest neighbors of $X$ can then be combined using TBM rule. We thus have

$$
m^{\Omega}\{C\}\left(\left\{w_{k}\right\}\right)=\left(1-\partial_{k}^{i}\right) \bigsqcup_{l \neq k} \partial_{l}^{i} \quad k=1, \ldots, m
$$

\section{B. Application KNN classifier to fault diagnosis of analog circuit using working temperate of analog circuit}

In area of fault diagnosis of circuit, if there are some faults components in the system, voltage values will deviate from the normal range. When some components are faulty, generally speaking, their temperatures will changed (no matter increase or decrease), then we can test the temperature of each component, and calculate the correlation coefficient. The proposed fault detection method using the KNN rule is based on the idea that the trajectory of a normal sample of working temperature of component in circuit is similar to the trajectories of normal samples in the training data; on the other hand, the trajectory of a fault sample of working temperature of component must exhibit some deviation from the trajectories of normal training samples. In other words:

- A fault sample's distance to the nearest neighboring training samples must be greater than a normal sample's distance to the nearest neighboring training sample.

- The distance between one type fault sample and the same type fault training sample must be smaller than any distance between the fault sample and normal sample in the training data and distance between this type of fault sample and other type of fault sample in the training data.

- The distance between normal sample and fault sample in training data also must be larger than the distance between normal sample and normal sample in the training data as well.

Based on this principle provided above, the steps of fault location by working temperature of component are shown as fellow:

- Model building: For each labeled sample $x_{i}$, $1 \leq i \leq n$, finding K-nearest neighbors for each sample in the training data set.

- Fault detection: For an incoming unclassified sample $\mathrm{x}$, finding $\mathrm{X}$ 's $\mathrm{K}$-nearest neighbors from the entire sample in training data set. 
- Calculation the X's KNN Euclidean distance $d$ between all the sample in the training data set and unclassified sample $\mathrm{x}$.

- Calculation all of $\partial_{q}^{i}$ and $m^{\Omega}\{C\}\left(\left\{w_{k}\right\}\right)$ using formula (3) $\sim(6)$ respectively.

- Comparison with each $m^{\Omega}\{C\}\left(\left\{w_{k}\right\}\right)$ and make a decision by pignistic.

\section{The Fault Diagnosis of the Analogue Circuit}

In this experiment, two example of fault diagnosis of analog circuit as shown in Fig. 2 are investigated using the proposed diagnosis method. In Fig.2, S2 Part indicates a lowpass filter with all components having standard tolerances. S1 Part indicates a band-pass filter with all components having standard tolerances which are shown in Fig 3.

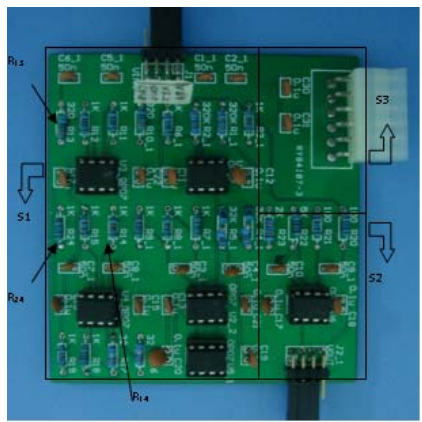

Fig.2 PCB under test

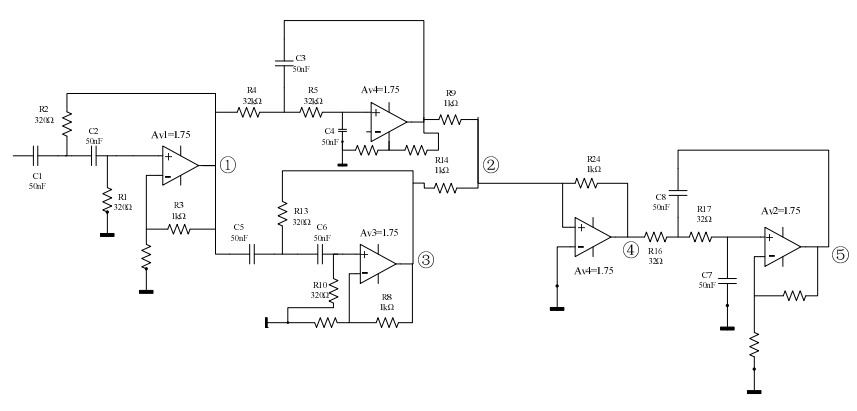

Fig.3 Band-pass filter circuit under test

The same fault diagnosis technique was then applied to a more complex circuit (see Fig.2 Part S1). The principle of this circuit is indicated in Fig.3. In this case, $R_{50 C}, R_{80 C}, R_{13}$, $R_{24}$ and $R_{14} \& R_{24}$ were considered to be potentially faulty elements. The circuit signature is constructed with the same procedure designed for the circuit. Considering the following faults.

- Training data:

Single faults:

Normal:

$---\mathrm{W}_{1}\left(\mathrm{R}_{5 \mathrm{OCC}}\right), \mathrm{W} 2\left(\mathrm{R}_{4 \mathrm{OC}}\right)$;

Soft faults:

$---\mathrm{W}_{3}$ (normal);

$---\mathrm{W}_{4}\left(\mathrm{R}_{24}\right), \mathrm{W}_{5}\left(\mathrm{R}_{13}\right)$;

$---\mathrm{W}_{6}\left(\mathrm{R}_{14}\right.$ and $\left.\mathrm{R}_{24}\right)$;

- Test data:

Test1: $\mathrm{R}_{13}$;Test2: $\mathrm{R}_{50 c}$;

Test3: $\mathrm{R}_{14} \& \mathrm{R}_{24}$;

Diagnosing the faults for the band-pass filter requires a neural network with five inputs, 16 first-layer and 5 output- layer neurons. The number of output neurons includes one for the no-fault class. This neural network also requires a training set of size 10 for each fault class under one frequent. To form the fault training data, the faulty component values have been extracted from the uniform distribution defined in the intervals $\left[0.1 X_{n} ;(1-t) X_{n}\right]$ and $\left[(1+t) X_{n} ; 10 X_{n} ;\right]$, where $t$ is the tolerance range and $X_{n}$ is the nominal value of the circuit element.

Our study of this circuit indicates that the neural network and KNN classification rule can't distinguish between the Normal and $\mathrm{R}_{4 \mathrm{OC}}$ fault classes. The reason Normal and $\mathrm{R}_{4 \mathrm{OC}}$ fault classes are indistinguishable is because they produce similar outputs. An inspection of this circuit reveals that these two fault classes have transfer functions in which corresponding terms are different by at most $6 \%$ assuming nominal values. As a result, based on features extracted from the output node, we can't separate Normal and $\mathrm{R}_{4 \mathrm{OC}}$ fault classes.

From the step mentioned in Section III Part B, the detail of the distance between Test2, Test 3 and each point of training group is provided in TABLE 1 . Using formula (6), we get $m^{\Omega}\{C\}\left(\left\{w_{k}\right\}\right)(k=1, \ldots, 6)$. The result is shown in TABLE 2. For example, using data from TABLE 1 and formula (6), we gets the results shown as follows:

$$
\begin{aligned}
\mathrm{m}^{\Omega}\{\text { test } 2\}\left[\left(\mathrm{w}_{6}\right)\right]= & (1-0.6178) \times 0.7244 \times 0.7790 \times \\
& 0.5793 \times 0.7244 \times 0.0268 \\
= & 0.0024
\end{aligned}
$$

The other data can get by the same method.

TABLE 1. ELCLIDEAN DISTANCE BETWEEN TEST2 AND EACH TEATURE SAMPLE

\begin{tabular}{|l|l|l|l|}
\hline Distance & $\mathbf{1 k h z}$ & Distance & $\mathbf{1 k h z}$ \\
\hline Test2 $\rightarrow \mathrm{W}_{1}$ & 0.0268 & Test3 $\rightarrow \mathrm{W}_{1}$ & 0.4932 \\
Test $2 \rightarrow \mathrm{W}_{2}$ & 0.7244 & Test3 $\rightarrow \mathrm{W}_{2}$ & 0.5847 \\
Test2 $\rightarrow \mathrm{W}_{3}$ & 0.5793 & Test3 $\rightarrow \mathrm{W}_{3}$ & 0.6252 \\
Test2 $\rightarrow \mathrm{W}_{4}$ & 0.7790 & Test3 $\rightarrow \mathrm{W}_{4}$ & 0.4317 \\
Test2 $\rightarrow \mathrm{W}_{5}$ & 0.7137 & Test3 $\rightarrow \mathrm{W}_{5}$ & 0.7074 \\
Test2 $\rightarrow \mathrm{W}_{6}$ & 0.6178 & Test3 $\rightarrow \mathrm{W}_{6}$ & 0.0590 \\
\hline
\end{tabular}

\begin{tabular}{|c|c|c|c|}
\hline$m^{\Omega}\{$ test 2$\}$ & $1 \mathrm{khz}$ & $m^{\Omega}\{$ test 3$\}$ & $1 \mathrm{khz}$ \\
\hline$m^{\Omega}\{\operatorname{test} 2\}\left[\left(w_{6}\right)\right]$ & 0.0024 & $m^{\Omega}\{$ test 3$\}\left[\left(w_{1}\right)\right]$ & 0.0033 \\
\hline$m^{\Omega}\{\operatorname{test} 2\}\left[\left(w_{2}\right)\right]$ & 0.0015 & $m^{\Omega}\{$ test 3$\}\left[\left(w_{2}\right)\right]$ & 0.0023 \\
\hline$m^{\Omega}\{\operatorname{test} 2\}\left[\left(w_{3}\right)\right]$ & 0.0028 & $m^{\Omega}\{$ test 3$\}\left[\left(w_{3}\right)\right]$ & 0.0019 \\
\hline$m^{\Omega}\{\operatorname{test} 2\}\left[\left(w_{4}\right)\right]$ & 0.0011 & $m^{\Omega}\{t e s t 3\}\left[\left(w_{4}\right)\right]$ & 0.0043 \\
\hline$m^{\Omega}\{$ test 2$\}\left[\left(w_{5}\right)\right]$ & 0.0015 & $m^{\Omega}\{$ test 3$\}\left[\left(w_{5}\right)\right]$ & 0.0013 \\
\hline$m^{\Omega}\{\operatorname{test} 2\}\left[\left(w_{1}\right)\right]$ & 0.1403 & $m^{\Omega}\{$ test 3$\}\left[\left(w_{6}\right)\right]$ & 0.0518 \\
\hline$M^{\Omega}\{\operatorname{test} 2\}[(\theta)]$ & 0.0039 & $M^{\Omega}\{\operatorname{test} 3\}[(\theta)]$ & 0.0032 \\
\hline $\begin{array}{l}\text { TABLE 3. NORMALIZ } \\
\text { INDUCE ON EA }\end{array}$ & \multicolumn{2}{|c|}{ INDUCE ON EACH FAULT SINGLETONS } & \\
\hline$m^{\Omega}\{$ test 2$\}$ & $1 \mathrm{khz}$ & $m^{\Omega}\{$ test 3$\}$ & $1 \mathrm{khz}$ \\
\hline
\end{tabular}

TABLE 2. PROBABILITY OF TEST2 AND TEST3 INDUCE ON EACH FAULT SINGLETONS 


\begin{tabular}{|c|c|c|c|}
\hline$m^{\Omega}\{$ test 2$\}\left[\left(w_{6}\right)\right]$ & 0.0156 & $m^{\Omega}\{$ test 3$\}\left[\left(w_{1}\right)\right]$ & 0.0485 \\
$m^{\Omega}\{$ test 2$\}\left[\left(w_{2}\right)\right]$ & 0.0098 & $m^{\Omega}\{$ test 3$\}\left[\left(w_{2}\right)\right]$ & 0.0338 \\
$m^{\Omega}\{$ test 2$\}\left[\left(w_{3}\right)\right]$ & 0.0182 & $m^{\Omega}\{$ test 3$\}\left[\left(w_{3}\right)\right]$ & 0.0279 \\
$m^{\Omega}\{$ test 2$\}\left[\left(w_{4}\right)\right]$ & 0.0072 & $m^{\Omega}\{$ test 3$\}\left[\left(w_{4}\right)\right]$ & 0.0632 \\
$m^{\Omega}\{$ test 2$\}\left[\left(w_{5}\right)\right]$ & 0.0098 & $m^{\Omega}\{$ test 3$\}\left[\left(w_{5}\right)\right]$ & 0.0191 \\
$M^{\Omega}\{$ test 2$\}[(\theta)]$ & 0.0254 & $M^{\Omega}\{$ test 3$\}[(\theta)]$ & 0.0470 \\
$m^{\Omega}\{$ test 2$\}\left[\left(w_{1}\right)\right]$ & $\mathbf{0 . 9 1 4 0}$ & $m^{\Omega}\{$ test 3$\}\left[\left(w_{6}\right)\right]$ & 0.7606 \\
\hline
\end{tabular}

TABLE 4. CLASSIFICATION RESULTS WITH PROPOSED METHOD

\begin{tabular}{|c|c|c|c|c|c|}
\hline $\begin{array}{c}\text { Fault } \\
\text { component }\end{array}$ & $\begin{array}{l}\text { Accuracy by low- } \\
\text { frequent neural } \\
\text { network (\%) }\end{array}$ & $\begin{array}{l}\text { Accuracy by high- } \\
\text { frequent neural } \\
\text { network }(\%)\end{array}$ & $\begin{array}{c}\text { Diagnosis } \\
\text { accuracy by KNN } \\
\text { classify }(\%)\end{array}$ & $\begin{array}{l}\text { Fusion diagnosing } \\
\text { results based on D- } \\
\text { S rule }(\%)\end{array}$ & $\begin{array}{c}\text { Fusion diagnosing } \\
\text { results based on } \\
\text { proposed method } \\
(\%)\end{array}$ \\
\hline $\mathrm{R}_{24}$ & $71.6 \%$ & $64.2 \%$ & $89.5 \%$ & $73.7 \%$ & $81.3 \%$ \\
\hline $\mathrm{R}_{13}$ & $79.7 \%$ & $50.7 \%$ & $92.3 \%$ & $69.8 \%$ & $78.9 \%$ \\
\hline $\mathrm{R}_{14} \& \mathrm{R}_{24}$ & $67.4 \%$ & $76.4 \%$ & $81.5 \%$ & $77.9 \%$ & $81.7 \%$ \\
\hline $\mathrm{R}_{5 \mathrm{OC}}$ & $70.1 \%$ & $64.5 \%$ & $95.7 \%$ & $73.7 \%$ & $89.5 \%$ \\
\hline
\end{tabular}

Table 3 shows the detailed performance of K-nearest neighbor classification rule in diagnosing hard fault $\mathrm{R}_{50 \mathrm{C}}$ and multi fault $\mathrm{R}_{14} \& \mathrm{R}_{24}$. Each column in this table corresponds to one fault class. Such result indicates the proposed KNN method can successfully identify the fault location. Comparing with BP network and KNN classification rule, K-nearest neighbor classification rule by component temperature is more effective than BP network by accessible node voltage if:

- The class of each fault in the learning set class is known precisely and categorically. For example, KNN classification rule is more effective when single hard fault happened for the reason that learning set class of hard fault is easier to be known and extracted precisely and categorically. Table 3 shows this result that the accuracy rate of hard fault diagnosis is much higher than soft fault.

- The learning sample can cover with all class of each fault in the learning set.

According to the formal fusion algorithm and KNN fault judgment rules based on component temperature, we can get final fusion diagnosis results shown in Table 4

Using the above fusion algorithm and fault judgment rules discussed in the previous section, we have studied the fault diagnosis of real sample circuits in Fig.7 and Fig.8. Table 4 shows the detailed performance of our fusion system in diagnosing the 5 fault classes associated with the five band-pass filter. Also in this case, a comparison among different methods is shown, a comparison among DempsterShafer methods is shown, and each row in this table corresponds to one fault class. Different columns then indicate the values of accuracy rate of diagnosis result. For example, the second and third columns of the table are respectively shows the rate of accuracy value of each components based on BP network by voltage sensors. The fifth and sixth columns show the accuracy of fault diagnosis after Dempster-Shafer evidence theory and transferable belief model.

Table 4 shows distinctly that the result by the single sensor can't identify the fault component correctly, but using fusion technique the synthetic diagnosis result can identify the fault component exactly. In the example, component $R_{13}$ is the real fault component, the diagnosis accuracy from BP network is $50.79 \%$, so we can't decide the fault component. But after transferable belief model fusion technique, the fault diagnosis accuracy increase significantly, and therefore we can point out the fault component correctly. In other words, the transferable belief model fusion algorithm makes the uncertainty of the diagnosis system decrease effectively, and error diagnosis has been removed from the inadequacy of single sensor information. This proves that our analog fault diagnostic system generalizes well and is capable of reliable performance using a small training set.

\section{ACKNOWLEDGMENT}

The aim of the paper is to present the potentials of the transferable belief model in fault diagnostic for analog circuit and to show its contribution to fault location performance. Diagnostic accuracy, stability, sensitivity and reliability are assessed in particular. Supporting diagnosing example is also obtained in this paper.

The results clearly confirm the superiority of the proposed approach over classical data fusion method and traditional techniques based on TBM framework. It is shown that TBM diagnostic method and K-nearest neighbor rule from TBM can significantly reduce the number of false location and is more effective than single sensor processing.

\section{REFERENCES}

[1] C. Alippi, M. Catelani, A. Fort et al., "Automated selection of test frequencies for fault diagnosis in analog electronic circuits," IEEE Transactions on Instrumentation and Measurement, vol. 54, no. 3, pp. 1033-1044, Jun, 2005.

[2] F. Aminian, M. Aminian, and H. W. Collins, "Analog fault diagnosis of actual circuits using neural networks," IEEE Transactions on Instrumentation and Measurement, vol. 51, no. 3, pp. 544-550, Jun, 2002.

[3] M. Catelani, and A. Fort, "Soft fault detection and isolation in analog circuits: Some results and a comparison between a fuzzy approach and radial basis function networks," IEEE Transactions on Instrumentation and Measurement, vol. 51, no. 2, pp. 196-202, Apr, 2002. 
[4] O. Basir, and X. H. Yuan, "Engine fault diagnosis based on multisensor information fusion using Dempster-Shafer evidence theory," Information Fusion, vol. 8, no. 4, pp. 379-386, Oct, 2007.

[5] X. F. Fan, and M. J. Zuo, "Fault diagnosis of machines based on D-S evidence theory. Part 1: D-S evidence theory and its improvement," Pattern Recognition Letters, vol. 27, no. 5, pp. 366-376, Apr, 2006.

[6] T. Denoeux, and P. Smets, "Classification using belief functions: Relationship between case-based and model-based approaches,” IEEE Transactions on Systems Man and Cybernetics Part B-Cybernetics, vol. 36, no. 6, pp. 1395-1406, Dec, 2006.
[7] P. Smets, "The combination of evidence in the transferable belief model," IEEE Transactions on Pattern Analysis and Machine Intelligence, vol. 12, no. 5, pp. 447-458, 1990

[8] P. Smets, "Analyzing the combination of conflicting belief functions," Information Fusion, vol. 8, no. 4, pp. 387-412, Oct, 2007.

[9] T. Denoeux, "A k-nearest neighbor classification rule based on dempster-shafer theory," IEEE Transactions on Systems Man and Cybernetics, vol. 25, no. 5, pp. 804-813, 1995. 